\title{
Avaliação morfológica e tensiométrica de pericárdio bovino pré-tratados em glutaraldeído e conservados em glicerina
}

\author{
[Morphological and tensiometrical evaluation of glutaraldehyde-treated bovine pericardium \\ preserved in glycerin]
}

\section{"Artigo Científico/Scientific Article"}

\author{
Diego Gonzalez Vivas ${ }^{1 *}$, José Vinicius Rodrigues Lopes ${ }^{1}$, Alessandra Santos Feijó da Silva \\ Souza $^{2}$, Lys Sirelli ${ }^{3}$, Viviane Alves Escócio ${ }^{3}$, Elen Beatriz Acordi Vasques Pacheco ${ }^{3}$, Vivian de \\ Assunção Nogueira ${ }^{4}$, Marta Fernanda Albuquerque da Silva ${ }^{1}$
}

\begin{abstract}
${ }^{1}$ Departamento de Cirurgia e Medicina Veterinária, Universidade Estácio de Sá, Rio de Janeiro-RJ, Brasil. ${ }^{2}$ Instituto de Ciência e Tecnologia em Biomodelos, Fundação Oswaldo Cruz (FIOCRUZ), Rio de Janeiro-RJ, Brasil. ${ }^{3}$ Instituto de Macromoléculas Eloísa Mano, Universidade Federal do Rio de Janeiro, Rio de Janeiro-RJ, Brasil. ${ }^{4}$ Departamento de Epidemiologia e Saúde Pública, Universidade Federal Rural do Rio de Janeiro, Rio de Janeiro-RJ, Brasil.

*Autor para correspondência/Corresponding author: E-mail: diegogonzalezvivas@gmail.com
\end{abstract}

\section{Resumo}

O presente estudo teve por objetivo descrever o comportamento morfológico e mecânico do pericárdio bovino tratado em glutaraldeído nas concentrações de $0,625 \%, 0,8 \%$ e 1,0\% por 18 dias, com posterior conservação em glicerina a $98 \%$ por 30 dias. Os pericárdios foram divididos nos grupos experimentais em controle (5 fragmentos de pericárdios bovinos conservados unicamente em glicerina a $98 \%$ por 30 dias seguidos), I ( 5 fragmentos de pericárdios bovinos tratados com glutaraldeído a $0,625 \%$ por 18 dias com posterior conservação em glicerina a $98 \%$ por 30 dias seguidos), II (5 fragmentos de pericárdios bovinos tratados com glutaraldeído a $0,8 \%$ por 18 dias com posterior conservação em glicerina a $98 \%$ por 30 dias seguidos) e III (5 fragmentos de pericárdios bovinos tratados com glutaraldeído a $1,0 \%$ por 18 dias com posterior conservação em glicerina a 98\% por 30 dias seguidos), para avaliação histológica; para os ensaios de tração, além dos grupos já descritos, foi adicionado o grupo in natura (5 fragmentos de pericárdio bovino recém obtido sem nenhum tipo de tratamento ou conservação). Foram observadas alterações no aspecto físico quanto à coloração e textura das membranas tratadas com glutaraldeído comparadas ao do grupo controle. Não houve alterações histológicas e mecânicas do pericárdio bovino do grupo controle para os grupos experimentais I, II e III, mostrando, portanto, que a associação do glutaraldeído com a glicerina para preservação do pericárdio bovino apresentou morfologia e um comportamento dinâmico semelhante ao do grupo controle, sendo assim, considerado satisfatório para uma possível aplicação clínica.

Palavras-chave: membranas biológicas; glicerol; glutaral.

\begin{abstract}
The present study aims to describe the morphological and mechanical behavior of bovine pericardium treated with glutaraldehyde for 18 days with subsequent preservation in glycerin for 30 days. The pericardium was divided into the experimental groups in control ( 5 fragments of bovine pericardium preserved only in glycerin for 30 consecutive days), I ( 5 fragments of bovine pericardium treated with $0.625 \%$ glutaraldehyde for 18 days with subsequent preservation in glycerin for 30 consecutive days), II (5 fragments of bovine pericardium treated with $0.8 \%$ glutaraldehyde for 18 days with subsequent preservation in glycerin for 30 consecutive days), and III (5 fragments of bovine pericardium treated with $1.0 \%$ glutaraldehyde for 18 days with subsequent preservation in glycerin for 30 consecutive days) for histological evaluation. For the traction tests, in addition to the groups already described, an in natura group (5 fragments of fresh bovine pericardium recently obtained without any type of treatment or conservation) was added. We observed changes in physical
\end{abstract}


appearance for coloration and texture of glutaraldehyde-treated membranes compared to the control group. There was no histological and tensiometric test abnormality comparing the pericardium in the control group to experimental groups I, II and III, showing therefore that the combination of glutaraldehyde with glycerin for preservation of the bovine pericardium showed morphology and dynamic behavior similar to the control group, so satisfactory for a possible clinical application.

Keywords: biological membranes; glycerol; glutaral.

\section{Introdução}

A utilização de membranas biológicas como material de implante para a reparação de órgãos e tecidos vem sendo praticada na medicina humana e veterinária. $\mathrm{O}$ seu emprego deve-se, principalmente, à facilidade de sua obtenção, baixo custo, preparo simples, fácil estocagem e pouca ou nenhuma reação tecidual. Grande parte dos trabalhos envolvendo membranas biológicas busca avaliar a viabilidade em procedimentos cirúrgicos nas diferentes regiões do organismo animal. $\mathrm{O}$ pericárdio é uma das membranas mais comumente estudadas, por possuir como característica principal a constituição quase exclusiva de colágeno (Girotto et al., 2003; Quitzan et al., 2003).

A reconstrução cirúrgica com utilização de membranas biológicas ainda é um desafio no sentido da padronização de biomateriais, metodologia de tratamento e conservação, tipos e concentração de soluções, biocompatibilidade, adequação à finalidade cirúrgica, dentre outras questões a serem uniformizadas. $\mathrm{O}$ meio de tratamento e conservação de membranas mais utilizado em medicina veterinária é a glicerina a 98\% em temperatura ambiente, que apresenta como vantagem o baixo custo e propriedade antisséptica, porém apresenta baixa ação antimicrobiana (Coronado et al., 2000; Quitzan et al., 2003). Os tratamentos tradicionais do pericárdio bovino na medicina humana $\mathrm{e}$ veterinária, quais sejam, associação de glutaraldeído $0,5 \%$ ou $0,625 \%$ a formaldeído $4 \%$, e glicerina $98 \%$ como solução única, deixam a desejar pelo alto nível de toxidez ou pela baixa efetividade antimicrobiana, respectivamente (Pinto et al., 1993; Martins et al., 2011; Costa et al., 2016).

As concentrações de glutaraldeído a $0,5 \%$ e $0,625 \%$, são consideradas esterilizantes e não prejudiciais à incorporação do implante. No entanto, alguns esporos de bactérias e vírus de incubação lenta não foram afetados pelo tratamento com estas concentrações (Hilbert et al., 1988). O aumento nas concentrações do glutaraldeído poderia eliminar os diferentes agentes infecciosos, entretanto, estas provocam elevada toxicidade no local do implante (Costa et al., 2016).

Visando a utilização dos bio implantes conservados e tratados numa solução ideal e em concentrações que não causassem interferências no processo de reparo tecidual e possíveis reações inflamatórias locais, Martins et al. (2011) e Costa et al. (2016) descreveram a utilização experimental de pericárdios bovinos pré-tratados por glutaraldeído nas concentrações de $0,625 \%, 1,0 \%$ e $1,5 \%$ e conservados em glicerina em parede abdominal em camundongos, os quais concluíram que os conservantes nas concentrações de $0,625 \%$ e $1,0 \%$ não produziram lesões macro ou microscópicas significantes que causassem efeitos deletérios e que não houve efeito tóxico direto desses conservantes sobre o fígado, baço e rim desses animais.

O conhecimento sobre a resistência das membranas biológicas conservadas é uma característica importante quando se planeja sua implantação para substituição de áreas das paredes corporais ou de vísceras ocas. Na avaliação histológica de membranas conservadas é possível observar-se alterações no padrão de organização tecidual, que influenciam na sua resistência. A avaliação mecânica é um método que vem sendo usado para estudo das características físicas das membranas biológicas após tratamento (Guimarães et al., 2007).

Conforme descrito por Martins et al. (2011) e Costa et al. (2016), que demonstram a possibilidade do pré-tratamento com glutaraldeído até $1,0 \%$ visando sua utilização mais segura em relação à transmissão de patógenos, mantendo as características de baixo custo e praticidade do uso e conservação dessas membranas em relação à utilização tradicional da glicerina como solução única de tratamento e conservação; o presente estudo teve como objetivo descrever o comportamento morfológico e mecânico de pericárdios bovinos pré-tratados em diferentes concentrações de glutaraldeído e conservados em glicerina a $98 \%$. 


\section{Material e Métodos}

Foram obtidos oito pericárdios de bovinos, com idade entre 30 a 36 meses, mestiços, machos e fêmeas, colhidos após o abate, em matadouro com Fiscalização Estadual na cidade de Três Rios - RJ. Para a conservação e transporte do material durante as duas horas de deslocamento, foi utilizada uma embalagem plástica nova mantida em recipiente térmico com gelo, mantendo-se os pericárdios em temperatura de aproximadamente $10^{\circ} \mathrm{C}$ até a chegada ao laboratório. Em prosseguimento, foi realizada a limpeza pericárdica, retirando-se a gordura utilizando uma pinça anatômica de dissecção e uma tesoura de Metzembaum reta para que o seu tratamento e conservação fossem iniciados. Os grupos experimentais foram divididos em grupo $\mathrm{G}$ (controle) - 5 fragmentos de pericárdios bovinos conservados unicamente em glicerina a $98 \%$ por 30 dias seguidos; grupo I - 5 fragmentos de pericárdios bovinos tratados com glutaraldeído a $0,625 \%$ por 18 dias com posterior conservação em glicerina a $98 \%$ por 30 dias seguidos; grupo II - 5 fragmentos de pericárdios bovinos tratados com glutaraldeído a $0,8 \%$ por 18 dias com posterior conservação em glicerina a 98\% por 30 dias seguidos; grupo III -5 fragmentos de pericárdios bovinos tratados com glutaraldeído a $1,0 \%$ por 18 dias com posterior conservação em glicerina a $98 \%$ por 30 dias seguidos; grupo in natura - somente para a avaliação tensiométrica com 5 corpos de prova de pericárdio bovino recémadquirido, os quais foram limpos para remoção da gordura como descrito anteriormente e sem sofrer nenhum tipo de tratamento ou conservação química, hidratados em solução fisiológica estéril a $0,9 \%$, ficando assim em igual condição experimental aos demais grupos.

As soluções em questão foram elaboradas no laboratório do Departamento de Medicina e Cirurgia Veterinária da Universidade Federal Rural do Rio de Janeiro (UFRRJ). Foi produzida uma solução PBS (Phosphate-Bufferid Saline), composta por fosfato monopotássio, fosfato dissódico, cloreto de sódio e água destilada para o tamponamento das soluções de glicerina bidestilada $98 \%$ e de glutaraldeído $25 \%$ (Proquimios ${ }^{\circledR}$ ), sendo esta última solução diluída em água destilada até atingir as concentrações de $0,625 \%, 0,8 \%$ e $1,0 \%$, tamponada com solução PBS com pH de 7,4, assim como a glicerina $98 \%$.

Para a avaliação histológica, os pericárdios bovinos, após a limpeza, foram fracionados no sentido longitudinal (ápice/base) em partes com áreas equivalentes a $2,0 \mathrm{~cm}^{2}$ e, em seguida, colocado um fragmento em cada frasco estéril de solução com capacidade de $70 \mathrm{~mL}$, sendo cinco fragmentos para cada grupo experimental. No grupo controle (glicerina) os fragmentos permaneceram na solução por 30 dias seguidos; nos grupos I a III, o tratamento com glutaraldeído nas diferentes concentrações perdurou por 18 dias e, após este período, os pericárdios foram conservados em frascos de glicerina por mais 30 dias seguidos.

Todos os frascos, devidamente identificados, permaneceram armazenados em temperatura ambiente sob proteção da luz. Aspectos macroscópicos foram observados nos diferentes grupos após os períodos de tratamento específicos, comparando-se com a membrana in natura e também entre eles. As características avaliadas consistiram em coloração, textura e forma das membranas após o período de hidratação preconizado (20 minutos), como descrito por Rojo et al. (2009). A análise histológica foi realizada no Laboratório de Anatomia Patológica do Departamento de Epidemiologia e Saúde Pública da UFRRJ. Foram confeccionadas 20 amostras teciduais de pericárdios bovinos, as quais foram devidamente tratadas, conservadas e identificadas como descrito anteriormente. Após a retirada dos fragmentos de seus respectivos frascos, estes foram lavados em solução salina estéril e reidratados. Os fragmentos foram fixados em formaldeído a $10 \% \mathrm{e}$ blocados para realização dos cortes histológicos num micrótomo, obtendo-se cortes de $5 \mu \mathrm{m}$ com auxílio de navalhas descartáveis, que foram submetidos à coloração de hematoxilina e eosina (HE), sendo as fotos documentadas utilizando-se câmera digital (Axiocam 105 color, 5mp ${ }^{\circledR}$ ) acoplada ao microscópico óptico Zeiss ${ }^{\circledR}$.

Os fragmentos preparados para avaliação tensiométrica foram produzidos $\mathrm{com} 2,0 \mathrm{~cm}$ de largura e $10 \mathrm{~cm}$ de comprimento no sentido longitudinal (ápice/base), sendo colocados em frascos estéreis de solução seguindo-se o mesmo método descrito anteriormente, exceto para o grupo in natura, no qual não foi realizado nenhum tipo de tratamento ou conservação química. As espessuras dos pericárdios foram mensuradas utilizando-se um paquímetro, o qual revelou a medida de $0,8 \mathrm{~mm}$ em média. Cinco fragmentos foram produzidos para cada grupo experimental.

O ensaio mecânico de tração foi realizado no Laboratório de Macromoléculas Eloísa Mano na Universidade Federal do Rio de Janeiro (UFRJ). Os 
grupos experimentais foram os mesmos do ensaio histológico, porém com acréscimo do grupo in natura, constituído pelos corpos de prova de pericárdio bovino recém-adquirido sem nenhum tipo de tratamento ou conservação química, somente hidratada em solução fisiológica estéril a $0,9 \%$, totalizando assim 25 corpos de provas. No laboratório, o material foi devidamente retirado de seus respectivos frascos e lavado em solução salina estéril (cloreto de sódio a 0,9\%) para retirada dos resíduos de sua superfície, e reidratados por 20 minutos no mesmo tipo de solução. Em seguida, os cinco corpos de prova de cada grupo foram devidamente enumerados para uma correta comparação e identificação.

Os testes foram realizados na máquina universal de ensaio $\mathrm{AME}^{\circledR}$ (Técnica Industrial Oswaldo Filizola) com capacidade de $5 \mathrm{kN}$. A máquina possui interface direta a um microcomputador com o software DynaView Standard/Pro M, capaz de gerar gráficos da carga versus tempo para cada ensaio. No momento do ensaio, os corpos de prova sofreram uma pré-carga de 2 Newton durante 30 segundos, com o intuito de promover acomodação do sistema, evitando-se possíveis folgas no conjunto de máquinas, acessório e modelo ensaiado.

Em seguida, a pré-carga prosseguiu com velocidade pré-estabelecida de $10 \mathrm{~mm} / \mathrm{mim}$ iniciando o ensaio de tração até a ruptura da membrana. A carga aplicada foi registrada pelo software em intervalos regulares de tempo até o momento da ruptura da membrana. Os dados foram agrupados e descritos estatisticamente por meio de valores médios e desvio padrão; utilizou-se teste não paramétrico de Mann-Whitney com nível de significância igual a $5 \%(p \leq 0,05)$ adotado em todas as comparações dos grupos estudados.

\section{Resultados}

Alterações no aspecto físico das membranas foram observadas principalmente nos grupos tratados com glutaraldeído nas diferentes concentrações e posteriormente conservados em glicerina. Em comparação aos pericárdios bovinos in natura, houve mudança significativa na coloração, de branco leitoso para amarelo acastanhado. A textura macia das membranas a fresco mostrou-se pouco alterada pela conservação em glicerina, porém a adição do tratamento com o glutaraldeído conferiu aspecto "emborrachado" em todas as concentrações utilizadas, acompanhado de alteração na forma das membranas, que mostravam leve encurvamento das margens dos retalhos.

Todas as membranas analisadas histologicamente, tanto as conservadas por glicerina a $98 \%$ por 30 dias quanto as pré-tratados em glutaraldeído nas concentrações de 0,625\%, $0,8 \%$ e $1,0 \%$ por 18 dias para posterior conservação em glicerina por mais 30 dias, apresentaram a mesma constituição estrutural. Observou-se a presença de tecido conjuntivo denso não modelado com fibras pouco acidófilas dispostas longitudinalmente sem orientação fixa, bem próximas entre si com núcleos acentuadamente basófilos. Comparando-se o grupo controle (grupo G) com os grupos experimentais, não verificou diferenças histológicas e estruturais entre as amostras (Figura 1).

Nas análises tensiométricas, a ruptura dos corpos de prova ocorreu quase em sua totalidade na sua região central, embora em alguns exemplares tenha ocorrido próximo de uma de suas extremidades, modificando assim o ponto de ruptura para uma região mais distal. Foram obtidas e analisadas as seguintes propriedades mecânicas: força máxima de tração e tensão máxima.

A Figura 2 mostra um exemplo do comportamento de resposta à tração de um corpo de prova do pericárdio bovino, aplicada na direção uniaxial até a força que causou a ruptura representada em Newtons. Pela análise da figura, pode-se observar que à medida que o tempo passa e a força de tração aumenta, vai ocorrendo seu alongamento, o que pode ser visualizado ao longo do teste. $\mathrm{O}$ comportamento da membrana submetida à tração pode ser dividido em três partes, definidas na figura. Inicialmente, ocorre $o$ alongamento para pequenos valores de força (trecho 1). No trecho 2, ocorre um aumento considerável de força à medida que o corpo de prova se alonga mais intensamente. E por fim, no trecho 3, após a força máxima, as fibras da membrana se rompem gradualmente, neste instante a força decresce enquanto ocorre o aumento visual do alongamento do corpo de prova.

Das observações referentes aos testes físicos com o pericárdio bovino, constatou-se que a força máxima calculada foi em média $86,08 \mathrm{~N}$ para amostras in natura, 97,64N para os pericárdios conservados em glicerina a $98 \%$ por 30 dias, $146,2 \mathrm{~N}$ para os pericárdios que sofreram pré tratamento com glutaraldeído a $0,625 \%$ e conservação em glicerina por 30 dias, $101,96 \mathrm{~N}$ nas amostras tratadas a $0,8 \%$ de glutaraldeído e 
$119,36 \mathrm{~N}$ nas tratadas por glutaraldeído a $1,0 \%$ (Tabela 1).

A área utilizada no cálculo da tensão máxima para os grupos de pericárdios bovinos foi de $16 \mathrm{~mm}^{2}$. A tensão máxima média calculada nesta amostra foi de $5,38 \mathrm{~N} / \mathrm{mm}^{2}$ para o grupo in natura; $6,10 \mathrm{~N} / \mathrm{mm}^{2}$ para o grupo $\mathrm{G} ; 9,13 \mathrm{~N} / \mathrm{mm}^{2}$ para o grupo I, no grupo II a tensão máxima foi de $6,37 \mathrm{~N} / \mathrm{mm}^{2}$ e no grupo III observou-se tensão de $7,46 \mathrm{~N} / \mathrm{mm}^{2}$ (Tabela 1 e Figura 3 ).

A análise estatística pelo teste não paramétrico de Mann-Whitney não mostrou diferença significante para os valores de força máxima e de tensão máxima entre os diferentes grupos.
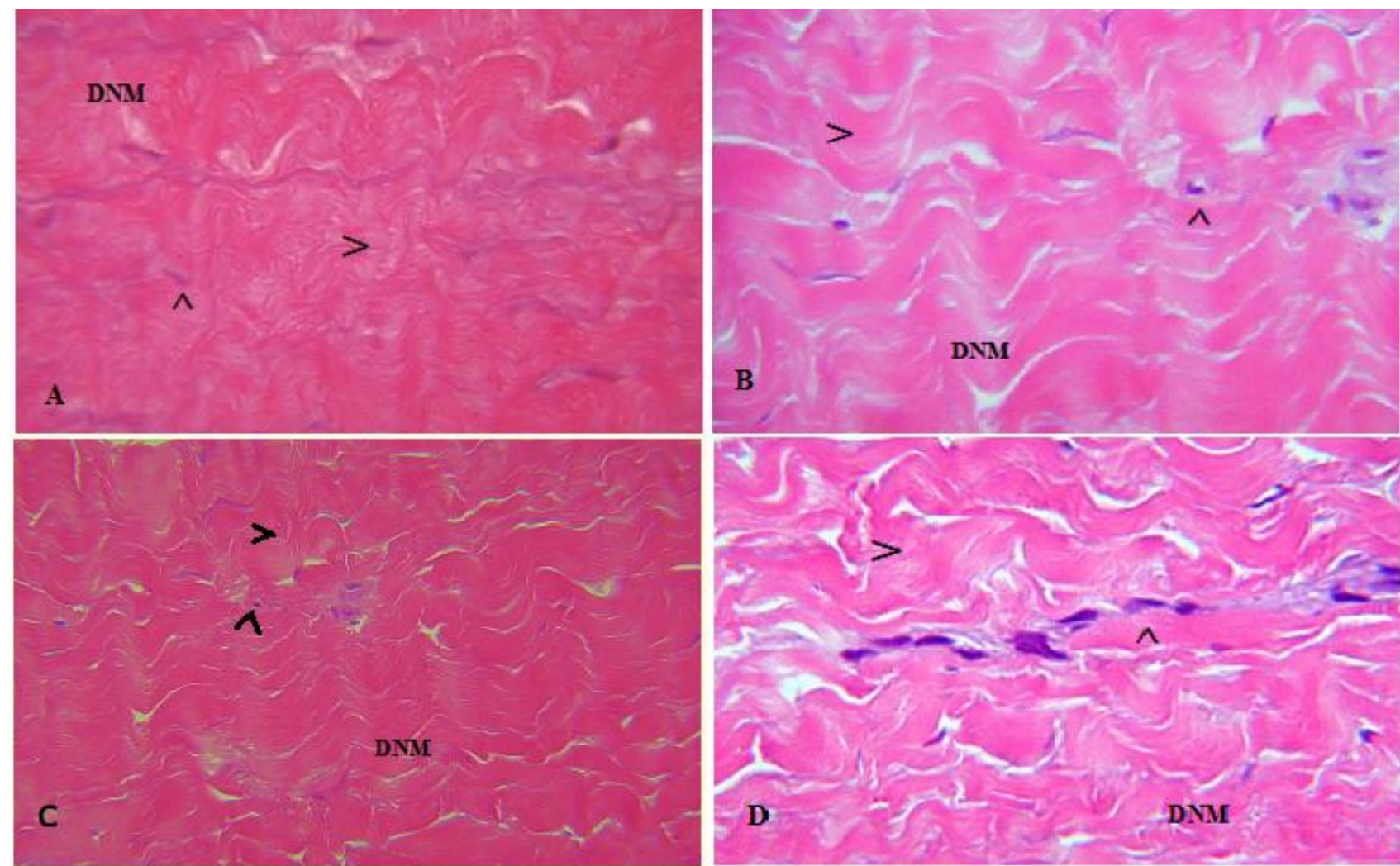

Figura 1. Fotomicrografias das amostras dos pericárdios bovinos na coloração HE. A. Grupo controle (aumento 40X). B. Grupo experimental I (aumento 40X). C. Grupo experimental II (aumento 40X). D. Grupo experimental III (aumento 40X). Nota-se que não há diferença histológica entre o grupo controle e os grupos experimentais, nos quais se observa tecido conjuntivo denso não modelado (DNM), fibras em disposição longitudinal (>) e núcleos acentuadamente basófilos $\left({ }^{\wedge}\right)$.

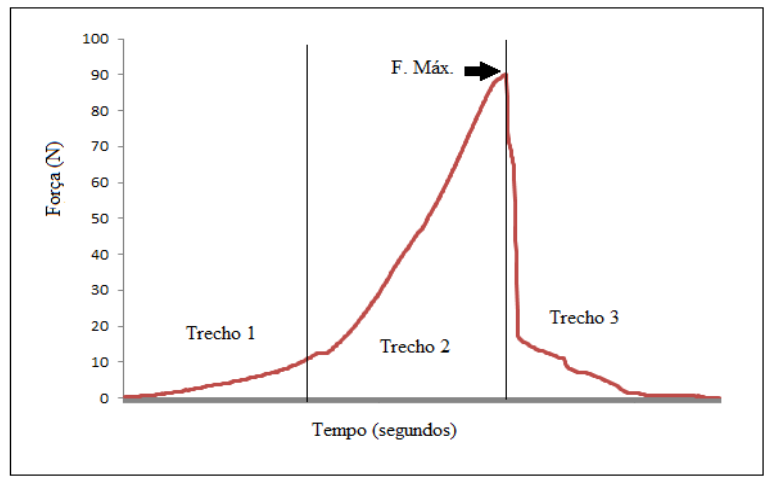

Figura 2. Comportamento força versus tempo durante um ensaio de resistência à tração de um corpo de prova de pericárdio bovino com aplicação de carga na direção uniaxial ao eixo longitudinal. Legenda: F. Máx. = Força Máxima.

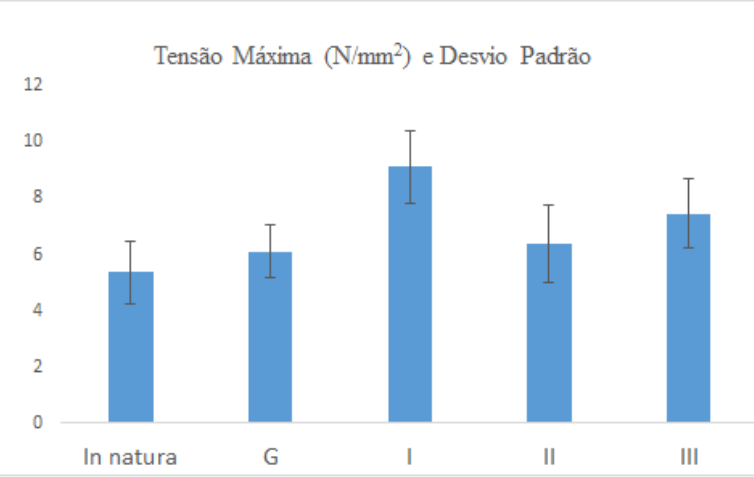

Figura 3. Representação gráfica dos valores médios da tensão máxima e desvio padrão dos corpos de prova dos pericárdios bovinos submetidos aos ensaios de tração nos diferentes grupos: in natura, grupo $\mathrm{G}$ (controle-glicerina 98\%) e grupos experimentais I (glutaraldeído $0,625 \%+$ glicerina $98 \%$ ), II (glutaraldeído $0,8 \%$ + glicerina 98\%), e III (glutaraldeído 1\% + glicerina 98\%). 
Tabela 1. Valores referentes à força máxima e tensão máxima dos fragmentos de pericárdio bovino submetidos ao ensaio mecânico de tração nos grupos in natura, grupo G (controle-glicerina 98\%) e os grupos experimentais I (glutaraldeído 0,625\% + glicerina 98\%), II (glutaraldeído 0,8\% + glicerina 98\%), e III (glutaraldeído 1\% + glicerina 98\%), expressos em valores médios \pm desvio padrão.

\begin{tabular}{cccccc}
\multirow{2}{*}{ Parâmetros } & \multicolumn{5}{c}{ Grupos experimentais } \\
\cline { 2 - 6 } & In natura & G & I & II & III \\
\hline Força Máxima (N) & $86,08 \pm 23,63$ & $97,64 \pm 33,32$ & $146,20 \pm 42,65$ & $101,96 \pm 30,69$ & $119,36 \pm 36,97$ \\
Tensão Máxima $\left(\mathrm{N} / \mathrm{mm}^{2}\right)$ & $5,38 \pm 1,11$ & $6,10 \pm 0,93$ & $9,13 \pm 1,29$ & $6,37 \pm 1,35$ & $7,46 \pm 1,23$ \\
\hline
\end{tabular}

\section{Discussão}

O pericárdio bovino foi obtido sem custos e com segurança em um abatedouro de inspeção estadual, comprovando-se os relatos de literatura que o consideram uma membrana de fácil aquisição (Costa et al., 2016). Pode-se afirmar também que o tratamento com glutaraldeído, assim como a manutenção em glicerina, é de fácil implementação, exigindo equipamentos simples e procedimentos de fácil execução, como descrito por Martins et al. (2011) e Costa et al. (2016).

A utilização da glicerina como conservadora de tecidos biológicos é difundida na medicina veterinária pelos seus efeitos antibacterianos, sendo questionada, entretanto, por sua ação antiviral (Coronado et al., 2000). É relatada, na maioria das vezes, como solução única no tratamento de várias membranas: peritônio bovino (Busnardo et al., 2009), pericárdio bovino (Quitzan et al., 2003; Miyamotto et al., 2009), pericárdio bovino em ratos (Martins et al., 2011; Collatusso et al., 2012; Costa et al., 2016), entre outras. O intuito de propor-se, no presente trabalho, o prétratamento dos pericárdios bovinos com $\mathrm{O}$ glutaraldeído, é o de possibilitar uma forma de utilização mais segura quanto à possibilidade de transmissão de patógenos, mantendo-se, entretanto, as características de baixo custo e praticidade do protocolo tradicional em nosso meio, que é a utilização da glicerina como solução única de tratamento e conservação.

Costa et al. (2016) utilizaram concentrações de glicerina e glutaraldeído semelhantes às utilizadas no presente experimento para tratamento de pericárdio bovino implantado em parede abdominal de camundongos, e verificou que concentrações de glutaraldeído acima de 1,5\% causam elevada toxicidade no local do implante, reforçando a segurança da utilização das concentrações entre $0,625 \%$ a $1,0 \%$ de glutaraldeído quanto à sua biocompatibilidade no tratamento de pericárdios bovinos. Martins et al.
(2011) observaram que estas mesmas biopróteses não provocaram lesões macroscópicas ou histológicas nos rins, fígado e baço dos 60 camundongos implantados. Estes resultados, oriundos de trabalhos do mesmo grupo de pesquisa do presente estudo, motivaram a determinação das concentrações de glutaraldeído aqui utilizadas.

Segundo Raiser et al. (2001), para a implantação cirúrgica de um biomaterial conservado em glicerina, este deve ser reidratado por um tempo mínimo, que varia de acordo com a densidade do material, pois a glicerina tem um poder de desidratação, dando-lhe assim sua característica conservadora. Para o presente estudo, os corpos de prova foram reidratados de acordo com Rojo et al. (2009), que utilizaram solução fisiológica a $0,9 \%$ em temperatura ambiente $\left(20^{\circ} \mathrm{C}\right)$ durante 20 minutos.

Os cortes de pericárdio bovino avaliados microscopicamente no presente trabalho mostraram o característico tecido conjuntivo denso não modelado (Junqueira e Carneiro, 2004) que constitui os revestimentos viscerais fibrosos. Este arranjo, apesar de característico da membrana pericárdica, não é uniforme em toda a sua extensão, conforme relatado por Rojo et al. (2009). Os autores estudaram a resistência de cortes de pericárdio bovino em dois diferentes sentidos, longitudinal (ápice/base) e circunferencial, observando que a resistência dos cortes circunferenciais é mais elevada, devido à disposição mais uniforme das fibras colágenas dos fragmentos obtidos desta forma; entretanto, os mesmos autores consideram que uma distribuição mais desordenada das fibras confere maior elasticidade aos fragmentos e, portanto, pode-se sugerir uma flexibilidade mais adequada à aplicação cirúrgica, que geralmente envolve a substituição de paredes corporais ou viscerais, as quais sofrem tensão em diversos sentidos. No presente estudo, os fragmentos foram produzidos, tanto para análise histológica quanto tensiométrica, 
no sentido longitudinal da membrana (ápice/base), visando uma avaliação de retalhos apropriados à utilização em cirurgias reconstrutoras.

O efeito do tratamento e conservação com glicerina sobre a estrutura morfológica das membranas biológicas vem sendo estudado ao longo do tempo e os trabalhos demonstram que não há influência desta substância sobre a organização tecidual. Em uma avaliação histológica de diversas membranas realizada por Guimarães et al. (2007), dentre as quais o pericárdio bovino, conservadas em glicerina a $98 \%$ por $15,30,60$ e 90 dias e a fresco, os autores não verificaram diferenças marcantes quanto à integridade estrutural dos pericárdios conservados em glicerina a $98 \%$ em comparação com o grupo controle (pericárdio a fresco). Nos três estudos, sugere-se que a glicerina possui uma ação inibitória enzimática impedindo a degeneração das fibras do material conservado, promovendo a preservação por uma ação desidratante, sem alteração das características da textura do tecido. Com base nestes dados da literatura, os pericárdios bovinos tratados com glicerina $98 \%$ foram considerados como grupo controle na avaliação morfológica do presente estudo.

Os resultados obtidos no presente estudo demonstram que o glutaraldeído também não causa alterações histológicas quando utilizado no prétratamento dos pericárdios bovinos nas concentrações de $0,625 \%, 0,8 \%$ e $1,0 \%$, comparando-se ao protocolo com a glicerina como solução única de tratamento e conservação.

Quanto às avaliações tensiométricas, os corpos de prova, ao serem submetidos à imposição da força axial externa de tração, sofreram estiramento e, consequentemente, alteração no valor da área em cada instante de aplicação da força, provocando, assim, a ruptura do fragmento na parte central da maioria dos corpos de prova exceto em alguns exemplares de pericárdio bovino que tiveram seu ponto de ruptura deslocado para a extremidade da amostra. A força máxima $(\mathrm{N})$ foi o principal parâmetro utilizado para comparar a diferença de resistência tecidual entre os grupos estudados, que representa a maior força aplicada durante o ensaio de tração.

Segundo Miyamotto et al. (2009), a forma de tratamento do pericárdio pode influenciar na sua resistência à ruptura, na ocasião os pericárdios foram tratados com glutaraldeído a $0,5 \%$ e conservados em formaldeído a 4\%. Os autores argumentam que o glutaraldeído estabelece ligações cruzadas entre os grupos aldeídos e amina das fibras colágenas, aumentando a estabilidade do tecido e, consequentemente, aumentando a resistência do material de forma mais eficaz que outras substâncias alternativas que podem ser utilizadas para este fim: difenilfosfarilazida e o etildimetilaminopropil carbodiamida. No presente estudo, os remendos de pericárdios pré-tratados em glutaraldeído nas concentrações de $0,625 \%, 0,8 \%$ e $1,0 \%$ por 18 dias para posterior conservação em glicerina a $98 \%$ por 30 dias, não tiveram sua resistência mecânica notavelmente alterada, visto pelos resultados de força máxima e tensão máxima dos pericárdios sem diferença estatística significante $(\mathrm{p}<0,05)$ quando comparados com os grupos in natura e glicerina. Alterações morfológicas macroscópicas foram observadas nos grupos pré-tratados com glutaraldeído, como mudanças na cor e textura das membranas, o que se esperava ser acompanhado por aumento na resistência mecânica, conforme relatado na literatura (Baucia et al., 2006). Sugere-se que a conservação em glicerina por 30 dias pode ter tido influência nos resultados tensiométricos do presente estudo, o que, entretanto, ainda precisa ser melhor examinado. Ainda, deve-se considerar que o aumento de resistência das membranas biológicas imputado ao glutaraldeído, que seria esperado, não é indispensável para sua indicação como solução de tratamento, uma vez que o pericárdio bovino conservado em glicerina $98 \%$ ou tratado com soluções de glutaraldeído nas concentrações estudadas já mostrou resistência adequada para seu uso clínico.

Vinculando-se as atividades antimicrobianas conhecidas do glutaraldeído e a praticidade da glicerina $98 \%$ na conservação de membranas biológicas, aos resultados aqui apresentados de compatibilidade destas soluções na manutenção das características morfológicas e mecânicas do pericárdio bovino, sugere-se que o método proposto é adequado para tratamento desta bioprótese.

\section{Conflito de interesse}

Os autores declaram não existir conflito de interesse.

\section{Comitê de Ética}

Os pericárdios bovinos foram obtidos após o abate, em matadouro com Fiscalização Estadual na cidade de Três Rios - RJ e, como não houve estudo experimental in vivo dessas membranas, não foi 
necessária a aquisição da licença à Comissão de Ética no Uso de Animais (CEUA).

\section{Referências}

Baucia, J.A.; Leal, R.M.; Rogero, J.R.; Nascimento, N. Tratamentos anticalcificantes do pericárdio bovino fixado com glutaraldeído; comparação e avaliação de possíveis efeitos sinérgicos. Revista Brasileira de Cirurgia Cardiovascular, 21(2): 180-187, 2006.

Busnardo, C.A.; Freitas, P.M.C.; Eurides, D.; Roncetti, G.R.; Nunes, L.C.; Beletti, M.E. Peritônio de bovino como bandagem de queimaduras cutâneas experimentais em coelhos. Revista Ciência Animal Brasileira, 10(3): 823-828, 2009.

Collatuso, C.; Roderjan, J.G.; Vieira, E.D.; Costa, F.D.A.; Noronha, L.; Fornazari, D.F. Efeito de descelularização com SDS na preservação da calcificação em pericárdio bovino fixado em glutaraldeído. Estudo em ratos. Revista Brasileira de Cirurgia Cardiovascular, 17(1): 88-96, 2012.

Coronado, G.S.; Swenson, C.L.; Martinez, S.A.; Burkhardt, K.S.; Arnoczky, S.P. Effects of a 98\% solution of glycerol or sterilization with ethylene oxide on FELV in bone allografts and effects on bone incorporation of allografts in cats. American Journal of Veterinary Research, 61(6): 665-671, 2000.

Costa, C.B.; Silva, M.F.A.; Andrade, G.B. Aspectos patológicos do implante de pericárdio bovino tratado pelo glutaraldeído e pela glicerina em parede abdominal de camundongos. Acta Scientiae Veterinariae, 44(1383): 1-7, 2016.

Gasque, K.C.; Oliveira, R.C.; Ceolin, D.; Cestari, T.M.; Taga, R.; Taga, E.M.; Correa, A. Avaliação da biocompatibilidade de uma membrana de pericárdio bovino acelular e seu potencial como carreador de osteoblasto. Ciência Odontológica Brasileira, 11(1): 5866, 2008.

Girotto; J.A.; Chicramonte, M.; Menon, N.G.; Singh, N.; Silverman, R.; Tufaro, A.P.; Nahabedian, M.; Goldbeg, N.H.; Manson, P. N. Recalcitrant abdominal wall hernias: long-term superiority of autologous tissue repair. Plastic and Reconstructive Surgery, 112: 106-114, 2003.

Guimarães, G.C.; Scavone, A.R.F.; Machado, M.R.F.; Cruz, C.; Capalbo, A.C.; Santos, A.L.Q. Avaliação histológica de membranas biológicas bovinas conservadas em glicerina e a fresco. Bioscience Journal, 23(3): 120-127, 2007.

Hilbert, S.L.; Ferrans, V.J.; Jones, M. Tissuederived biomaterials and their use in cardiovascular prosthetic devices. Medical Progress Through Technology, 14(3-4): 115163, 1988.

Junqueira, L.C.; Carneiro, J. Histologia básica. $10^{\mathrm{a}}$ ed. Rio de Janeiro: Guanabara Koogan, 2004. $487 \mathrm{p}$.

Martins, C.R.P.; Silva, M.F.A.; Nogueira, V.A.; Prado, J.S; Brito, M.F. Avaliação anatomopatológica de rins, fígado e baço de camundongo submetidos a implante de pericárdio bovino conservado em glicerina e glutaraldeído em ferida cirúrgica de parede abdominal. Revista Brasileira de Medicina Veterinária, 33(2): 103-110, 2011.

Miyamotto, M; Del Valle, C.E.; Moreira, R.C.R.; Timi, J.R.R. Resistência tensional do pericárdio bovino fixado em glutaraldeído comparado com a da veia safena magna. Jornal Vascular Brasileiro, 8(2): 103-111, 2009.

Pinto, T.J.A.; Saito, T.; Glerean, A. Biocompatibilidade de materiais empregados na confecção de próteses cardiovasculares: comparação entre pericárdio de bovino e Dacron. Revista de Saúde Pública, 27(3): 185189, 1993.

Quitzan, J.G.; Rahal, S.C.; Rocha, N.S.; Crocci, A.J. Comparação entre pericárdio bovino preservado em glicerina e malha de poliéster no reparo de falhas da parede abdominal em ratos. Acta Cirurgica Brasileira, 18(4): 297-301, 2003.

Raiser, A.G.; Graça, D.L.; Pippi, N.L.; Zinn, L.L.; Silveira, D.S.; Bordin, A.I.; Baiotto, G.C.; Rios, M.V.; Silveira, A.F. Homoimplante Ortopédico de tendão calcâneo em cães. Conservação, assepsia e implantação. Ciência Rural, 31(1): 89-94, 2001.

Rojo, F.J.; Atienza, J.M.; Jorge-Herrero, E.; Garcia-Páez, J.M.; Guinea, G.V. Resistencia a tracción de membranas de pericárdio para válvulas cardíacas biológicas. Anales de Mecanica de la Fractura, 1(26): 33-36, 2009. 